\section{Hacia un pensar colectivo. Una experiencia de extensión universitaria en las ruralidades uruguayas}

\author{
Mariana Quintana \\ emebuaca@gmail.com \\ Pablo Capurro \\ pacaqui@gmail.com
}

\author{
Daniel González Fajardo \\ dangonfaj@gmail.com \\ Universidad de la República, \\ Uruguay
}

Cultura(s) en clave de extensión universitaria / Intervenciones
RECEPCIÓN: 24/04/19

ACEPTACIÓN FINAL: 30/05/19

\section{Resumen}

El siguiente artículo relata una experiencia de trabajo acerca del fomento y promoción del asociativismo en la localidad de Paso Centurión (Cerro Largo), Uruguay. Dicho proyecto es fruto de la financiación de Proyectos Estudiantiles de Extensión Universitaria de la Comisión Sectorial de Extensión y Actividades en el Medio de la Universidad de la República.

El desarrollo de este proyecto se llevó a cabo en las modalidades de encuentros y talleres con la finalidad de discutir conceptualmente el asociativismo como forma de organización. Este trabajo fue realizado en conjunto con dos grupos de la zona e instituciones referentes en la temática y fue posible así identificar las potencialidades y los desafíos de estos emprendimientos en la zona. Sus resultados arrojan la necesidad de reflexionar sobre la estrategia del trabajo asociado en ámbitos rurales, el ecoturismo, las políticas de conservación ambiental y el rol de las instituciones públicas en el fortalecimiento de la autogestión.

Palabras clave: asociativismo rural, extensión, ecoturismo, conservación, autogestión.
Towards a collective thinking. An university extension experience in the uruguayan ruralities

\section{Abstract}

The following article relates an experience work on the promotion and promotion of associativism in the town of Paso Centurión (Cerro Largo), Uruguay. This project is the result of the financing of Student Projects of University Extension of the Sectorial Commission of Extension and Activities in the Middle of the University of the Republic. In the development of this project, we worked on the modalities of meetings and workshops, with the purpose of conceptually discussing associativism as an organizational form. This work was carried out in conjunction with two groups in the area and institutions that are relevant to the subject, being able to identify the potential and challenges of these ventures in the area. The results of this work show the need to reflect on the strategy of associated work in rural areas, ecotourism, environmental conservation policies and the role of public institutions in strengthening self-management.

Keywords: rural asociativism, extension, ecotourism, conservation, self-management.
Em direção a um pensar coletivo. Uma experiencia de extensão universitária nas ruralidades uruguaias

\section{Resumo}

$\mathrm{O}$ artigo a seguir relata um trabalho de experiência sobre o fomento e promoção do associativismo na cidade de Paso Centurión (Cerro Largo). Este projeto é o resultado do financiamento de Projetos Estudantis de Extensão Universitária da Comissão Setorial de Extensão e Atividades no Meio da Universidade da República.

No desenvolvimento deste projeto, trabalhamos nas modalidades de reuniões e workshops, com o objetivo de discutir conceitualmente o associativismo como uma forma organizacional. Este trabalho foi realizado em conjunto com dois grupos da área e instituições relevantes para o assunto, sendo capaz de identificar o potencial e os desafios desses empreendimentos na área. Os resultados deste trabalho mostram a necessidade de refletir sobre a estratégia de trabalho associado em áreas rurais, ecoturismo, políticas de conservação ambiental e o papel das instituições públicas no fortalecimento da autogestão.

Palavras-chave: associativismo rural, extensão, ecoturismo, conservação, auto-gestão.

Para citación de este artículo: Quintana, M.; Capurro, P. y González Fajardo, P. (2019). Hacia un pensar colectivo. Una experiencia de extensión universitaria en las ruralidades uruguayas.+E: Revista de Extensión Universitaria, 9(10), 108-123. doi: 10.14409/extension.v9i10.Ene-Jun.8321. 


\section{Introducción}

Los Proyectos Estudiantiles de Extensión Universitaria (PEEU) son fondos concursables de la Comisión Sectorial de Extensión y Actividades en el Medio (CSEAM) de la Universidad de la República (UdelaR) que buscan "fortalecer las prácticas de extensión universitaria, desarrolladas por colectivos estudiantiles, desde diversas disciplinas y en distintos puntos del país, enfatizando la formación y la dimensión pedagógica de los procesos de extensión universitaria" (UdelaR, s/f). La propuesta permite a los equipos de estudiantes contar con financiación y asesoramiento técnico para desarrollar las prácticas. Cada propuesta es acompañada por un docente orientador, pero quienes ejecutan el proyecto son los estudiantes, los cuales eligen voluntariamente presentar propuestas para llevar a cabo prácticas de extensión universitaria. Así, nuestro grupo, integrado por estudiantes de Psicología, Geografía, y nuestro docente referente con formación en Antropología y Ciencias Sociales, se postuló para dar continuidad al trabajo que se venía desarrollando en territorio. Este artículo relata la experiencia del grupo en la ejecución de una práctica extensionista que tuvo por objetivo estimular el asociativismo en el marco de los sistemas de protección ambiental en Paso Centurión, Cerro Largo.

Paso Centurión es una localidad en la zona rural del departamento de Cerro Largo, a 60 $\mathrm{km}$ de Melo, la capital departamental, a unos $400 \mathrm{~km}$ de Montevideo en dirección noreste. Como muchos de los pueblos de la ruralidad de Uruguay, se caracteriza por ser un poblado altamente envejecido, que estimamos no supera las 100 personas. En necesario contextualizar la manera en que se constituye la ruralidad en Uruguay con sus características tanto poblacionales como físicas y productivas. En Uruguay vive algo más de tres millones de personas, de las cuales la mitad habita en la capital y otro gran porcentaje en otras ciudades del país. Según el censo realizado por el Instituto Nacional de Estadística (en 2011), en ámbitos rurales de Uruguay viven menos de 180.000 personas, de las que un $55 \%$ son hombres y un $45 \%$ de mujeres. Casi 97.000 personas son menores de 45 años, y de ellas prácticamente hay mitad de hombres y mitad de mujeres. La franja de más de 45 años y hasta más de 100 años tiene una característica similar, con un pequeño incremento del porcentaje de mujeres en las edades más avanzadas.

En tanto, este poblado transita un contexto de cambios en los usos productivos del suelo, cambio que no es ajeno al proceso uruguayo suscitado a raíz del alza de los precios de commodities a nivel internacional. Particularmente destacamos el aumento de la actividad sojera y la forestal, amparado en la creación de instrumentos como las Leyes N $N^{\circ} 15.939$ de 1987 (“Ley Forestal") y № 16.906 de Promoción de Inversiones.

Estos cambios en los modelos productivos y de los productos exportados (frente a una tradición de producción ganadera) han permitido el ingreso de inversiones extranjeras alentadas por la estabilidad democrática del país y su austeridad fiscal. En este sentido, hay un nuevo proceso de concentración de la tierra debido a las formas productivas de estos commodities. A esto se suma la instalación de dos plantas de producción de pasta de celulosa en el país, lo que genera un crecimiento importante de la economía del Estado. Actualmente, el Estado uruguayo se ha comprometido con la empresa finlandesa UPM para la construcción de una tercera planta cerca de la zona de Paso de los Toros. Es así que la actividad forestal ha crecido exponencialmente y provocado una alta concentración de tierras, con poca mano de obra para su usufructo y altamente tecnificada. Este cambio genera que la población rural asala- 
riada no reúna los requisitos para el ingreso al mercado laboral forestal y pierda puestos de empleo, dado que la producción forestal reduce el espacio de la producción ganadera (actividad mayoritaria de la producción agropecuaria del Uruguay, sobre todo en el norte del país).

Por otra parte, se creó en el año 2000 el Sistema Nacional de Áreas Protegidas (SNAP), adecuándose a las normativas internacionales y apuntando a la conservación de ecosistemas específicos como últimos reductos de la fauna y flora nativa del país. Se ha delimitado una serie de áreas que se definen casi exclusivamente por criterios biológicos, y en ellas se espera generar la "integración del desarrollo productivo con la conservación de los recursos naturales"(MVOTMA, s/f), siendo la población estimulada a la producción sustentable y la promoción del turismo. Paso Centurión y Sierra de Ríos están en proceso de ingreso al SNAP debido a sus particularidades ecosistémicas. Como manifiesta el propio organismo en su "Plan Estratégico del SNAP 2015-2020": "la inclusión de Paso Centurión - Sierra de Ríos aportaría a la conservación de la biodiversidad de Uruguay priorizada por el SNAP 1 ecorregión, 5 ecosistemas amenazados y 37 especies amenazadas SNAP (prioritarias para el SNAP)" (2018:14).

Esta área tiene una extensión de 62.889 ha y está compuesta por dos ecorregiones: Sierras del Este y la Cuenca Sedimentaria Gondwánica. El proceso de ingreso de Paso Centurión y Sierra de Ríos al SNAP ha sido apoyado por una población que ve amenazadas sus oportunidades en la zona. Parte de los factores está relacionada a la falta de oportunidades laborales y educativas, así como al crecimiento de la actividad forestal en la zona y el alza de los precios de la tierra asociados a esta actividad productiva. Por lo tanto, para muchos pobladores el ingreso al SNAP significa una oportunidad de seguir viviendo en el lugar y una forma de defensa ante el cambio de las lógicas productivas intensivas de gran escala.

Con la llegada de los gobiernos progresistas a Uruguay (Castro y Santos, 2018), a partir de 2005 se estimuló a emprendimientos asociativos como forma de dinamizar las economías locales y evitar la pérdida de puestos de trabajo con el cierre de algunas empresas. Se crearon herramientas como el Fondo para el Desarrollo (FONDES) y se modificaron las formas de acceso a tierras del Instituto Nacional de Colonización (INC), ente que gestiona las tierras del Estado. En este escenario, los procesos asociativos rurales han tenido una transformación en los últimos 12 años e incorporaron la tenencia de la tierra a colectivos de asalariados y pequeños productores, en contraposición a la práctica histórica de tenencia de particulares o instituciones agrarias. El surgimiento de un colectivo para la explotación de tierras de modo asociativo en una zona tradicionalmente de explotaciones individuales significa un cambio en las ideas y las respuestas que elabora la comunidad para hacer frente a las necesidades de subsistencia. De esta manera, en Paso Centurión podemos encontrar dos emprendimientos asociativos, uno vinculado al INC, con el acceso a un campo ubicado en la zona, y otro colectivo enfocado a servicios de turismo. En el diseño de las políticas de estímulo al asociativismo se prevé un acompañamiento técnico para la transferencia de tecnologías para el trabajo asociado. En González Fajardo (2016) podemos ver cómo las formas de asesoramiento técnico están más orientadas hacia la aplicación de tecnologías para el funcionamiento grupal que a la producción de una identidad grupal que permita fortalecer la visión del asociativismo como estilo de vida.

En este contexto hemos ejecutado, en 2018, un Proyecto Estudiantil de Extensión Universitaria (PEEU) financiado por CSEAM para el trabajo con los emprendimientos asociativos de 
la zona en la construcción de una perspectiva local del asociativismo, entendiendo que una comunidad organizada tiene mejor capacidad de respuesta ante los intereses de los privados en la construcción de áreas de conservación.

\section{Pensar el turismo y el asociativismo en las ruralidades uruguayas}

La construcción de las territorialidades en los ámbitos rurales en general está asociada a las modalidades productivas de cada zona. Entendemos que:

"El territorio es sinónimo de apropiación, de subjetivación fichada sobre sí misma. Él es un conjunto de representaciones las cuales van a desembocar, pragmáticamente, en una serie de comportamientos, inversiones, en tiempos y espacios sociales, culturales, estéticos, cognitivos". (Guattari y Rolnik, 1986:323, en Haesbaert, 2004)

En esta línea de pensamiento, se observa la generación de territorios sobre la tierra y a su vez cartografías del conjunto de representaciones que las instituciones van delimitando. Para Nuñez:

"territorio es, casi literalmente, toda planicie, toda extensión de tierra y todos los objetos que se colocan sobre ella (inmuebles, semovientes, árboles, personas) sobre los que se aplica la mecánica de un poder militar o policiaco: el poder clásico. (...) Es un poder que observa objetos: vigila, mide, controla, calcula, registra, censa, cuenta y describe". (2014:13)

En esta construcción institucional de los territorios existen varias categorizaciones, como la descripción de grupos de suelos de la Comisión Nacional de Estudio Agronómico de la Tierra (CONEAT) y las Regiones Agroeconómicas (Griffin, 1972) y su propuesta de reformulación (Domínguez, et al., 2017). A este respecto, la zona de Cerro Largo ha sido caracterizada como un territorio dividido entre lo forestal-ganadero y lo ganadero-arrocero, con suelos CONEAT de baja productividad, donde predominan los suelos superficiales, con pendientes más o menos pronunciadas propias del paisaje de sierras.

A su vez, existe una caracterización de la población rural creada para identificar los sectores económicos y las dinámicas de inserción laboral. Para ello utilizamos los aportes de Chiappe, Carámbula y Fernández (2008) y Piñeiro (2002), como modelo para la caracterización. En estos trabajos se definen tres categorías: asalariados, productores familiares y empresarios rurales.

En la tipificación de Chiappe, la principal característica de los empresarios capitalistas es que explotan su producción fundamentalmente a través del empleo de mano de obra asalariada. Participen en la producción o no, el trabajo asalariado siempre es predominante. Dentro de aquellos, los empresarios ganaderos se caracterizan por priorizar la posesión de la tierra frente a la realización de inversiones, con uso extensivo de este recurso y sistemas tradicionales de explotación con escasa incorporación de mejoras (Chiappe et al., 2008:75).

Por otra parte, el productor familiar se considera "como aquel productor pobre, con pocos recursos productivos (poca tierra, maquinaria escasa y vieja, poco capital) y bastante tradicional (reticente a la adopción de tecnología)" (p. 83). Así, predomina el trabajo familiar (en propiedad o en usufructo) sobre la tierra. Mientras que parte de la producción se destina al autoconsumo, la mayoría se vuelca en mercados formales, distinguiéndose por esta característica del campesinado. 
En cambio, los asalariados rurales son concebidos como "todos aquellos trabajadores que lo hacen por un salario, sea este en forma mensualizada, jornalizada, a destajo o según cualquier forma de pago" (p. 103). En cuanto a la relación laboral según rubros productivos, la permanente se da más en la ganadería y en el rubro forestación es de tipo temporal.

\section{Etapas del asociativismo en Uruguay}

Existen diversos estudios que destacan la historia de los procesos asociativos en Uruguay desde principios del siglo XX. Entre estos trabajos, destacamos el de Terra (1986) sobre el desarrollo histórico y de significación de los procesos asociativos, con su posterior complemento de Isola y Martí (2015). Parte de esta historia está muy vinculada al desarrollo del cooperativismo, el cual tiene una fuerza muy particular, sobre todo con relación a la vivienda. Dado este contexto histórico, se utiliza el término asociativismo para no dejar afuera otras formas de trabajo asociado que no son necesariamente cooperativas. En este sentido, entendemos al asociativismo como "el proceso por el cual una o más personas y/o grupo(s) deciden reunirse de forma regular, pero no necesariamente continua, para atender demandas comunes" (Peixoto de Albuquerque, 2004a:31). Esta caracterización resulta lo suficientemente amplia como para involucrar la diversidad de procesos de trabajo asociado en Uruguay, incluyendo el cooperativismo y otras formas de hacer frente a esas "demandas comunes". También consideramos, al igual que Sarachu (2009), que un emprendimiento puede ser asociativo en tanto todos sus integrantes compartan algunos aspectos fundamentales. Estos aspectos a ser compartidos son: el trabajo, la información sobre el emprendimiento, los resultados económicos, el poder de decisión y los medios de producción. Cuanto más compartidas sean estas responsabilidades y más compromiso haya en la tarea a realizar, los emprendimientos tienen mayores posibilidades de sostenibilidad. Asimismo, creemos que para poder compartir estos componentes se hace necesaria la autogestión, entendida como "un modo del actuar colectivo, según el cual los principios de la acción social se forman en la experiencia concreta y provienen del significado dado a las intenciones o las ideas que fundamentan el grupo" ( Peixoto de Albuquerque, 2004b:46).

En cuanto al asociativismo en las ruralidades uruguayas, proponemos tres etapas que comprenden un primer momento experimental, donde surgen los primeros antecedentes del asociativismo rural en Uruguay; una primera etapa, donde las experiencias alcanzan una madurez mayor que termina con la llegada de la dictadura militar (1973); y una etapa contemporánea que surge luego del retorno a la democracia. Ver Cuadro 1.

En la primera etapa propiamente dicha, los emprendimientos asociativos logran un nivel más alto de formalización. Esto, junto con el apoyo de los gobiernos desarrollistas de la época, logra consolidar una serie de leyes que potenciarán la estrategia asociativa como forma de hacer frente a necesidades comunes de la población. A su vez, a esta etapa se suma un componente ideológico que ve a la organización de los sectores rurales como un camino para la transformación teniendo como referencia la Revolución Rusa y las reformas agrarias que se venían reclamando en Latinoamérica. En Uruguay se retoman ideales del artiguismo, como el "Reglamento de Tierras de 1815" (principal antecedente de la creación del INC). Esta etapa termina con la asunción del gobierno de facto y la persecución de todas aquellas instituciones que estaban basadas en la cooperación de sus socios, a las cuales se tildó de comunistas. 
Con el retorno de la democracia se tuvieron que conjugar estrategias para hacer frente a las crisis producidas por las transformaciones impulsadas por los gobiernos neoliberales de la época. Por lo tanto, existe un crecimiento importante del sector privado, en el cual se incluyen los emprendimientos asociativos. En ese entonces se apuesta a que el sector asociativo haga frente a las necesidades comunes, que con el "achique del Estado" y la política de no-intervención ya no tendrían respuesta estatal. Esta etapa incluso puede tener una subetapa a partir de la llegada de los gobiernos progresistas. Si bien se sigue apostando al sector privado como principal dinamizador de la economía, la intervención del Estado pasa por una mayor recaudación fiscal y la búsqueda de financiaciones internacionales (sobre todo las del Banco Interamericano de Desarrollo). En este marco se busca crear políticas públicas que fortalezcan el asociativismo a partir de transferencias monetarias y tecnológicas.

En la última etapa comienzan a aparecer estímulos para la vinculación entre asociativismo, conservación, producción y turismo.

Cuadro 1. Etapas del asociativismo en Uruguay.

\begin{tabular}{|c|c|c|c|c|c|}
\hline \multicolumn{2}{|c|}{ Primeros antecedentes } & \multicolumn{2}{|c|}{ Primera etapa } & \multicolumn{2}{|c|}{ Etapa contemporánea } \\
\hline 1871 & $\begin{array}{l}\text { Asociación Rural del } \\
\text { Uruguay }\end{array}$ & 1941 & $\begin{array}{l}\text { Ley de cooperativas } \\
\text { agrarias }\end{array}$ & 1980 & $\begin{array}{l}\text { Federación Nacional de } \\
\text { Asalariados Rurales }\end{array}$ \\
\hline 1915 & $\begin{array}{l}\text { Comisión Nacional de } \\
\text { Fomento Rural }\end{array}$ & 1948 & Ley de Colonización & 1994 & $\begin{array}{l}\text { Asociación de Mujeres } \\
\text { Rurales }\end{array}$ \\
\hline \multirow[t]{3}{*}{1935} & $\begin{array}{l}\text { Cooperativa Nacional de } \\
\text { Productores de Leche } \\
\text { (CONAPROLE) }\end{array}$ & 1950 & Comunidad del Sur & 2005 & Programa Uruguay Rural \\
\hline & & 1960 & Cooperativa Cololó & 2010 & Cambio en el perfil de \\
\hline & & 1961 & $\begin{array}{l}\text { Unión de Trabajadores } \\
\text { Azucareros de Artigas }\end{array}$ & & tierras del INC \\
\hline
\end{tabular}

Fuente: elaboración propia, 2018.

\section{El turismo como nuevo producto en las ruralidades}

El turismo rural nace en Europa a mediados del siglo pasado en países como Francia o España, que desde la recuperación económica y con un aumento en la demanda turística comienzan a promocionar las ruralidades como una alternativa a los clásicos destinos turísticos (Mendivil, 2009). La propuesta en concreto es crear una oferta turística donde el público pueda disfrutar de un entorno natural, vida al aire libre, y participar de las actividades típicas de la zona.

En América Latina surge el turismo rural como una forma de complementar los ingresos de las familias productoras que, a partir de la crisis económica que se suscita en la década de los ' 90 , deben diversificar su actividad. Es un intento por parte de las comunidades de ofrecer un servicio que complemente los ingresos a los hogares y resignifique el lugar de las mujeres y jóvenes, siendo una actividad que genera fuentes de empleo (en un contexto donde la inserción laboral de las mujeres y jóvenes es muy baja). Con el espíritu de brindar oportunidades de trabajo en emprendimientos que sean amigables con el medio ambiente, el 
SNAP considera que el ecoturismo o turismo rural es una oportunidad para las áreas protegidas, para dar mayor visibilidad a los lugares más conservados en cuanto a sus ecosistemas. Entendemos, como Mendivil, que el turismo rural

"se concibe como una vía hacia la gestión de todos los recursos, de forma que puedan satisfacerse las necesidades económicas, sociales y estéticas, respetando al mismo tiempo la integridad cultural, los procesos ecológicos esenciales, la diversidad biológica y los sistemas que sostienen la vida". (2009:4)

\section{Extensión en la UdelaR}

La extensión universitaria de la UdelaR deriva de los ideales forjados en el Movimiento de Reforma Universitaria Latinoamericana de Córdoba, donde las tres funciones de la universidad son la enseñanza, la investigación y la extensión. Posteriormente, en la UdelaR se transita por una segunda reforma al considerar necesario que las funciones de la universidad acompañen las necesidades, transformaciones y desafíos que se dan a nivel social, cultural y económico. Esto se evidencia con la creación de las comisiones sectoriales (Enseñanza, Investigación y Extensión), encargadas de dinamizar dichas funciones en la UdelaR. Desde la CSEAM se generan también diversas unidades temáticas que apuntan a fortalecer ciertas áreas de interés. Estas áreas se vinculan con el territorio a través de proyectos para abordar problemáticas y características de cada población. Una de ellas es la Unidad de Estudios Cooperativos (UEC), especializada en estudios sobre el asociativismo, el cooperativismo y la economía social y solidaria. La UEC "Contribuye al estudio y a la formación académica en la materia, para todo cual coordina los necesarios apoyos y asesoramientos técnicos que se requieran" (UdelaR-CSEAM, 2019).

En esta línea, y con relación al trabajo llevado adelante por la UEC-CSEAM, hemos adoptado una noción de extensión como herramienta para el trabajo universitario, colaborativa con los diferentes actores sociales y sus territorios, con necesidades y diversidad de saberes que se articulan para construir colectivamente formas de participación y de abordar las problemáticas.

Desde la experiencia concreta, la extensión universitaria es un aula-práctica en territorio. Para todas aquellas/os que participamos, se encuentra abierta al devenir y a la transformación. En cuanto a sus aspectos metodológicos, se apuesta a la experimentación del acierto y error, a la construcción del vínculo, al trabajo horizontal y, de igual forma, a la construcción de saberes enriquecidos por la diversidad de entendimientos sobre el hacer y cómo producirlo.

\section{Estrategia de intervención}

En la construcción de proyectos, Pichon-Rivière (2010) dice que existen al menos tres etapas por las cuales los grupos humanos transitan. Una primera instancia es poner en cuestión los cambios que experimenta un sujeto dentro de un grupo. Esta etapa está caracterizada por ansiedades y miedos propios de la resistencia al cambio. En todo proyecto (y más en uno asociativo) se ponen en cuestión los pareceres individuales y se entra en una etapa de negación o fragmentación entre la realidad y los imaginarios. Es por esto que en esta etapa de pretarea se buscan vías rápidas de escape, conflictos o pequeñas distracciones que nos 
alejan de la tarea. En cuanto esta última, nos dice: "En el pasaje de la pretarea a la tarea, el sujeto efectúa un salto, es decir, previa sumación cuantitativa de insight realiza un salto cualitativo durante el cual se personifica y establece una relación con el otro diferenciado" (2010:35). Es decir, el momento de la tarea es cuando finalmente podemos trabajar sobre las ansiedades de la pretarea, enfocándonos en el proyecto desde una postura global que nos devuelve al centro de la pretarea y comienza el nuevo espiral ascendente hacia la tarea. Por lo tanto, uno de los enfoques estratégicos principales de nuestra intervención consiste en simplificar el estadio de pretarea aportando a que el grupo mantenga su atención enfocada en la tarea y el proyecto. Esta modalidad de intervención es sinérgica y no apunta a eliminar las ansiedades sino a hacer cada vez más compleja la proyección y la tarea y, por consiguiente, las incertidumbres.

En este proyecto de intervención nos hemos propuesto estimular el asociativismo en el marco de los sistemas de protección ambiental en Paso Centurión, Cerro Largo. Este objetivo se desglosa en algunos objetivos específicos que se relacionan con: fortalecer la experiencias ya existentes en materia de asociativismo en la zona; estimular la creación de nuevos procesos asociativos como pueden ser cooperativas, sociedades de fomento rural u otro tipo organizaciones civiles; y generar encuentros con referentes nacionales en materia de asociativismo y los pobladores de la zona, con la finalidad de generar un debate sobre las posibilidades del asociativismo en Paso Centurión.

Hemos desarrollado tres encuentros que lograron abarcar los intereses construidos con la población. Asimismo, para facilitar el análisis de esta experiencia, la hemos sistematizado a través de tres ejes que transversalizan los aprendizajes que surgen de la práctica. Estos tres ejes son: lo vivencial, lo interinstitucional y lo conceptual. Consideramos que en todo fortalecimiento grupal, en especial de emprendimientos autogestionados, es necesario poder trabajar al menos estos tres puntos, los cuales creemos imprescindibles para la sostenibilidad de los mismos.

\section{Los grupos en Paso Centurión}

En este apartado nos proponemos introducir al lector en los emprendimientos asociativos con los cuales hemos trabajado a lo largo del proyecto (ver Cuadro 2). Estos emprendimientos cuentan con historias y objetivos distintos pero comparten el territorio y las formas organizativas. A continuación, presentamos brevemente, uno a uno, los emprendimientos asociativos que desarrollan sus actividades en Paso Centurión.

\section{Grupo Colonos de Centurión}

Este grupo está integrado por 9 personas (todos hombres) que han accedido en 2017 a un campo del INC a través de un llamado público. El INC realiza estos llamados con campos que son comprados o enajenados para luego darlos en arrendamiento a grupos de productores familiares con dificultades de escala o necesidad de capitalización. De esta forma, el INC pretende apoyar a las poblaciones más desfavorecidas en las ruralidades uruguayas con relación al acceso a la tierra. Este es el caso del Grupo de Centurión, que se dedica a la actividad ganadera, principalmente bovina, y que cuenta con asesoramiento técnico mediante financiaciones del propio INC. Es importante destacar que, si bien todos son vecinos de la 
zona, estos no tienen experiencia de trabajo en conjunto y crearon el grupo para acceder al llamado que era específico para grupos. Esto no significa una novedad en cuanto a la conformación de grupos en las ruralidades uruguayas. El INC adjudica en arrendamiento tierras por debajo del valor del mercado privado y por lo tanto las personas ven como una oportunidad presentarse a un llamado público para grupos que los gestionen. Así se conforman aglomerados de familias con necesidad de tierras que devienen en grupos. Este es el caso del Grupo Centurión, que está compuesto por productores pequeños y/o asalariados con necesidad de crecer en capital productivo.

\section{Grupo de Vecinos de Centurión}

Este colectivo está conformado por vecinas y vecinos de Paso Centurión que presentaron en 2017 un proyecto de turismo comunitario y ecoturismo a PPD (Proyecto de Pequeñas Donaciones) del Ministerio de Turismo (MINTUR), llamado "Vecinos de Centurión por una oferta ecoturística en pos del empoderamiento local". El emprendimiento ha contado con talleres de formación específica para el desempeño de las diversas tareas (senderismo, primeros auxilios, asociativismo rural, entre otros). Además, se ha producido el intercambio con otros colectivos que se dedican al mismo rubro y compras de insumos. En el colectivo participan 4 personas ( 3 mujeres y 1 hombre), pero incluye también a vecinas y vecinos que cuentan con predios donde se realizan los senderos y comercios de la zona. Esto favorece la capacidad de tender redes vecinales solidarias a través del emprendimiento, generar estabilidad frente a la falta de empleos remunerados (especialmente para mujeres y jóvenes) y el despoblamiento en la zona.

\section{JULANA}

Jugando en la Naturaleza (JULANA) es una ONG integrada principalmente por biólogos y biólogas orientados hacia la educación ambiental. El colectivo utiliza herramientas lúdicopedagógicas para abordar las relaciones entre sociedad y naturaleza, apostando a la participación activa de las poblaciones objetivo. Actualmente trabaja en Paso Centurión y en la Quebrada de los Cuervos con proyectos específicos para cada área. En Paso Centurión, uno de los proyectos es de apoyo al "Grupo de Vecinos de Centurión" a través de asesoramiento para la presentación y gestión del PPD. A su vez, parte de los integrantes de la ONG participan en la realización del Espacio de Formación Integral (EFI, UdelaR) que se lleva a cabo allí desde 2013. Otro de los ámbitos de desarrollo en la zona se asocia con el monitoreo participativo de fauna por medio de la instalación de cámaras trampa. De esta manera, JULANA es un grupo de referencia en la zona tanto para vecinos como para el ámbito universitario.

Cuadro 2. Esquema comparativo de grupos.

\begin{tabular}{|c|c|c|c|}
\hline Grupo & Origen & Objetivo & Integración \\
\hline Grupo de Colonos & $\begin{array}{l}\text { Productores familiares y asalariados de Paso } \\
\text { Centurión sin tenencia de tierras }\end{array}$ & Producción ganadera & $\begin{array}{l}\text { Productores familiares y asalariados, } \\
\text { todos son hombres. }\end{array}$ \\
\hline Grupo de Ecoturismo & Grupo de vecinas y vecinos de Paso Centurión & Servicio de turismo & Principalmente integrado por mujeres. \\
\hline JULANA & ONG de Montevideo & Educación ambiental & $\begin{array}{l}\text { Profesionales, principalmente de for- } \\
\text { mación en ciencias biológicas. }\end{array}$ \\
\hline
\end{tabular}

Fuente: elaboración propia, 2019. 


\section{Hacia un fortalecimiento sostenible del asociativismo}

En el trabajo de promoción del asociativismo en el territorio nos propusimos abordar tres ejes que aportan a la sostenibilidad de los proyectos. Sabemos, a través de la caracterización de estos emprendimientos, que la sostenibilidad, esto es, las posibilidades de subsistencia del proyecto, son parte de las tensiones iniciales que deben ser abordadas en los primeros años. Con el fin de poder dar herramientas para la sostenibilidad, configuramos dichos ejes, que son: lo vivencial, lo interinstitucional y lo conceptual. Para el abordaje de estas tres temáticas hemos desarrollado propuestas metodológicas que nos permitieron acercarnos a las realidades y tensiones de cada grupo. Asimismo, en cada uno de estos ejes hemos rescatado valiosos aprendizajes en el intercambio con los grupos que consideramos de gran valor y un aporte para el trabajo con grupos desde la extensión universitaria.

\section{Lo vivencial: pasear antes de trabajar}

El primer eje de trabajo es lo vivencial, entendiéndolo como sinónimo de experimentación personal. Un proyecto colectivo, asociativo, siempre requiere de cierta dosis de impacto personal, ya que estos emprendimientos transforman la cotidianidad de sus participantes e involucran anhelos de modificación de la realidad. La construcción de un proyecto colectivo exige cierto alineamiento de los proyectos individuales y familiares (Migliaro y Picos, 2008). Por lo tanto, fomentar la experiencia compartida y realizar actividades recreativas entre los participantes ayuda a la buena convivencia, a instaurar un clima de confianza y conocimiento de la vida de los pares. Conocer las aspiraciones y la trayectoria vital de los participantes, así como lo que está en juego para ellos en el proyecto, es fundamental para pensar el lugar que ocupa dentro de la organización. De modo que nos hemos propuesto como primera actividad abordar lo vivencial con el plus de poder ir a conocer otro emprendimiento de similares características. Como zonas de desarrollo próximo (Riviere, 1984), los intercambios generan la posibilidad de lograr un cierto grado de insight que refleje las potencialidades y tensiones de estos emprendimientos. Conocer otras experiencias nos ayuda a aprender cosas nuevas y a saber más sobre uno mismo.

Es así que, en el marco de las actividades propuestas en el proyecto PPD, acompañamos al grupo de vecinos y vecinas de Centurión a una visita al emprendimiento asociativo de ecoturismo que se desarrolla en la localidad de Laureles, departamento de Tacuarembó.

En esta ocasión definimos algunos aspectos a relevar, como las articulaciones para el trabajo asociativo, las formas organizativas, la distribución de tareas y la toma de decisiones y roles dentro del colectivo. También se procuró identificar los fenómenos de articulación entre proyectos (sean asociativos o no) que se desarrollan en el territorio, como una respuesta a las dificultades propias de las ruralidades y como posibilitadores del establecimiento de redes de apoyo, necesarias para la sostenibilidad de los proyectos.

El grupo de desarrollo Laureles tiene una historia de 12 años. En sus inicios fue integrado por 22 miembros, de los cuales dos familias son las únicas que ofrecen los servicios de ecoturismo en la actualidad. En cuanto a la estrategia de comunicación del emprendimiento, realizan contactos por correo electrónico y Facebook, aunque destacan que el "boca a boca" es el principal medio de difusión del proyecto.

La propuesta de ecoturismo cuenta con servicio de alojamiento y senderos elaborados por el grupo en varios predios, tanto de propiedad de los integrantes del grupo como de otros 
vecinos. Los distintos alojamientos se encuentran en los establecimientos de los productores integrantes del grupo que complementan su actividad ganadera con la de ecoturismo. Existe un alojamiento (propiedad de la Iglesia) en comodato con el grupo que anteriormente funcionó durante tres años como Escuela Técnica (UTU-ANEP).

Los establecimientos de Bichadero, Refugio la Gruta y La Casona son los tres alojamientos que están funcionando en la actualidad. La recepción de los visitantes se gestiona de forma individual por parte de cada establecimiento, y en los casos en que se sobrepasa la capacidad de un establecimiento, se derivan a los alojamientos disponibles. El arancel que se cobra incluye todos los servicios (alojamiento, comida y paseos). Además, incorporan algunos productos como parte de la oferta: licores, dulces y conservas. Existe una lógica colaborativa entre los alojamientos que también se utiliza para las visitas guiadas, donde pueden oficiar de guías los distintos integrantes del grupo.

Algunos de los resultados de este encuentro se asocian con la experiencia de intercambio entre los emprendimientos, donde un grupo que recién comienza adquiere nuevas herramientas fruto de la trayectoria de otro. Consideramos que el encuentro generó un impulso en el grupo al ver el funcionamiento de otro similar, reforzando así las expectativas y las certezas acerca del emprendimiento propio. Esto se retoma más adelante en otras instancias de trabajo con el grupo, en las cuales nos confesaron que no les gustaba la idea de "salir a pasear antes que trabajar" (ya que el emprendimiento aun no funcionaba para la recepción de turistas), pero luego se dieron cuenta de que era una buena oportunidad para aprender.

Otra de las actividades que se consideran dentro de lo vivencial es la recorrida de los senderos que el grupo de turismo construyó. Estos senderos sirvieron como excusa para largas conversaciones y para que el grupo pueda practicar su discurso de guías. A su vez, permite ir valorando los paisajes a través de la mirada y la afectación del otro. Todas estas actividades han contribuido a afianzar el vínculo y la confianza con el grupo, habilitando a sumergirse en el ámbito interpersonal.

\section{Lo interinstitucional: desafíos y perspectivas del asociativismo en la zona de Paso Centurión}

Después de la visita a Laureles se realizó una instancia general pensada para toda la comunidad. Esta jornada tuvo por objetivo generar el encuentro entre vecinos, emprendimientos asociativos de la zona, colectivos que trabajan en territorio e instituciones referentes en la temática del asociativismo. Entendemos que para lograr una sostenibilidad real para emprendimientos que se encuentran tan aislados es necesaria una red interinstitucional que brinde un soporte a la organización. Es por esto que se invitó a instituciones que consideramos de referencia en la temática para poder hacer efectivo el intercambio y algunos acuerdos de trabajo. La jornada fue planificada en cinco momentos. En el comienzo se realizó una presentación general de todos los participantes. Luego se presentó el proyecto estudiantil y se introdujo acerca de los componentes más relevantes del trabajo en la zona.

Se prosiguió con la presentación de experiencias asociativas de la zona: el proyecto PPD (ecoturismo) "Vecinos de Centurión", el Grupo de Colonos de Centurión y la ONG JULANA. Después las instituciones participantes dieron cuenta de la perspectiva de asociativismo que promueven, su forma de trabajo en el territorio y su visión de las posibilidades de desarrollo para la zona. 
El encuentro-taller reunió a 25 personas de diferentes colectivos e instituciones, ${ }^{1}$ y allí se pudieron generar los primeros intercambios. Además, cada persona e institución y/o colectivo pudo transmitir cómo trabaja y qué objetivos persigue. Esta presentación ofició de puntapié inicial para que en espacios subgrupales se identificaran los desafíos y las potencialidades del trabajo asociativo así como para conocer de primera mano la necesidad de apoyo por parte de las instituciones participantes.

En la identificación de potencialidades del trabajo asociativo surgieron temáticas diversas. Se destacó la grupalidad, la importancia de ser locales y la posibilidad de generar nuevos recursos de subsistencia que propongan una alternativa al despoblamiento en la zona. De esta manera, se rescató la riqueza del territorio más allá del uso productivo convencional que se le da a la tierra, a lo que se le suma valor paisajístico y cultural como otros recursos a ser tenidos en cuenta para la oferta de servicios. Aquí cobra protagonismo el emprendimiento de ecoturismo. También dentro de las posibilidades del asociativismo, se destacó el trabajo en conjunto, el hecho de compartir el interés por las temáticas ambientales, la socialización de la información, la importancia de la participación de los espacios de gestión de proyectos, promover habilidades de comunicación dentro de los grupos (escuchar, participar y disfrutar) y de vinculación con instituciones referentes. Se hizo foco asimismo en que, dentro de la participación en emprendimientos asociativos, se convive con la diversidad de puntos de vista, lo que produce diferentes formas de habitarlos. Esto se identifica como un desafío para dichos emprendimientos. Otros desafíos planteados para el trabajo de los grupos fueron: la articulación con los organismos del Estado y entre los proyectos existentes en la zona, mejorar estas articulaciones y contribuir al desarrollo de los emprendimientos.

Con respecto al cierre de la jornada, Gerardo Sarachu, de la Unidad de Estudios Cooperativos (UEC-CSEAM, UdelaR), desarrolló los cinco aspectos fundamentales (Antonio Cruz, 2007) a ser tenidos en cuenta por los emprendimientos asociativos haciendo referencia: al trabajo, a la propiedad de los medios, el poder de decisión, a la información y a los resultados económicos. A partir de esta referencia teórica y el alto interés planteado por los/las participantes de los emprendimientos, se abrió la posibilidad y el desafío de trabajar sobre la base de esos cinco aspectos en otra instancia. Esto propició un giro a nivel del marco teórico del proyecto y la reconfiguración de la propuesta de trabajo.

\section{Lo conceptual: hacia un pensar colectivo}

Otra de las instancias se relacionó con la necesidad de realizar un trabajo conceptual para poder intercambiar ideas y construir una idea común de asociativismo. Esta instancia surgió a solicitud de JULANA a través del proyecto PPD. En dicho proyecto se propone generar una capacitación para los integrantes del colectivo teniendo como primer módulo la profundización sobre herramientas para el trabajo asociativo. Este apartado responde a la sistematización de dicho encuentro, el cual se realizó en dos jornadas. El primer día se reflexionó acerca del concepto de asociativismo, además hacer una breve introducción histórica del asociativismo en las ruralidades uruguayas y los emprendimientos asociativos de turismo en ámbitos rurales (dificultades y aspectos a fortalecer). 
Para eso utilizamos las caracterizaciones definidas en el libro La Otra Economía (Peixoto de Albuquerque, 2004a), específicamente el apartado de asociativismo, las cuales se fueron presentando y discutiendo punto por punto. Los aspectos relacionados con la historia del asociativismo en Uruguay fueron tomados de clases de la UEC sobre asociativismo rural y los correspondientes a emprendimientos asociativos de turismo en ámbitos rurales fueron tomados de Mendivil (2009) y Szmulewicz, Gutiérrez y Winkler (2012).

En la segunda jornada se retomó el encargo de trabajar los cinco puntos propuestos por la UEC, que son aquellos aspectos necesarios a compartir. Fueron distribuidos en un "gráfico de radar" o "tela de araña" donde cada vértice representaba uno de estos aspectos. También se realizaron tarjetas basadas en las afirmaciones de Peixoto de Albuquerque (2004b) que resaltaban aspectos positivos y negativos de los emprendimientos autogestionados. En primer lugar, se leyeron las tarjetas y se pusieron a consideración del grupo. Si el contenido no era representativo del grupo, se excluía la tarjeta. En el caso de que el contenido sí estuviera relacionado con el sentir del grupo, se pasaba a discutir: ¿con cuál de los puntos se identifica más la frase? ¿Qué zona del gráfico debe ocupar la tarjeta? Algunas tensiones que se visualizaron en el diagrama fueron: la concentración en los resultados, teniendo en cuenta que es un emprendimiento joven y muchas de las ansiedades (pretarea) se depositan allí. Identificamos que no solo se refieren a resultados económicos sino a otros como: resoluciones que se encuentran pendientes y acceso a un lugar específico donde poder recepcionar a las/los turistas.

En otra lectura del gráfico, la concentración estaba en el centro, donde muchas de las frases disparadoras fueron identificadas por las/los participantes en conexión con varios aspectos al mismo tiempo. La evaluación de este taller se considera exitosa ya que se logró reflexionar sobre la situación actual del proyecto y la necesidad de pensar el emprendimiento a largo plazo (una de las debilidades de los emprendimientos asociativos). Además, se consiguió incorporar conceptos del asociativismo así como cuestionar las propuestas y estrategias aportadas por los autores. Por último, cabe destacar la novedad de la técnica utilizada, la cual fue creada específicamente para esta instancia pero hemos logrado reproducirla con otros emprendimientos de manera exitosa. Esta modalidad de gráfico permite identificar los principales nudos problemáticos a partir de frases genéricas que podrían estar relacionadas con cualquiera de los puntos colocados en el gráfico. La concentración o dispersión de las tarjetas puede analizarse en conjunto con los participantes y así exteriorizar las tensiones.

\section{Consideraciones finales}

La concreción de cada una de las instancias comentadas y su balance son altamente positivos, puesto que las líneas de trabajo pautadas tuvieron los resultados buscados. Logramos trabajar los aspectos relacionados con el asociativismo a través de la experiencia de los colectivos, del contacto con las políticas públicas y con algunos actores del asociativismo en las ruralidades uruguayas. Consideramos que estos emprendimientos son la respuesta de las comunidades a las dificultades que su propio medio plantea, donde el trabajo en la construcción de redes de apoyo es de suma importancia para los grupos. La UdelaR, y en particular los servicios de extensión universitaria, habilitan el desarrollo de articulaciones y otras actividades que son una apuesta a la transformación de las realidades con las poblaciones más desfavorecidas. 
En cuanto a nuestro objetivo de poder estimular el asociativismo en la zona, se logró identificar al menos dos posturas claras respecto de esta forma de trabajo. Existe, por un lado, una mirada escéptica y desconfiada acerca de las posibilidades de estos emprendimientos en lo que hace a su durabilidad, sostenibilidad económica y el aporte a la transformación de sus realidades. Esta mirada se asocia principalmente a pobladores envejecidos, que no participan en espacios colectivos y que tienen sus propios emprendimientos. $Y$ estos aspectos se condicen con la caracterización propuesta por Chiappe, Carámbula y Fernández (2008), entendiendo que la lógica de división del trabajo responde a necesidades de subsistencia diferentes y, por lo tanto, estrategias diferentes. Por otro lado, las juventudes y las familias que dependen del trabajo asalariado ven a estos emprendimientos como una posibilidad de permanencia en el territorio y de mejorar sus condiciones de vida. De todas maneras, estas experiencias representan un desafío para comunidades que históricamente han desarrollado modelos de producción individual. Por lo tanto, la universidad puede desplegar un rol articulador entre las políticas y las necesidades de los emprendimientos y fortalecer así sus capacidades y el diálogo de saberes.

Algunas de las discusiones que se suscitaron en el desarrollo de este trabajo se relacionan con las políticas de conservación ambiental. Las mismas producen desafíos en los modelos productivos actuales, por los cuales se deben transformar las estrategias de subsistencia e incorporar nuevas prácticas y tecnologías. Como hemos mencionado anteriormente, desde nuestra perspectiva, el trabajo asociado y la conformación de espacios colectivos, una comunidad organizada, generan mayores posibilidades de respuesta a intereses externos. Entendemos que para lograr una óptima estrategia de conservación es necesario profundizar en las acciones que habilitan a la participación. A su vez, es preciso que las poblaciones cuenten con oportunidades de subsistencia adaptadas a sus necesidades de permanencia y expresión cultural.

Las poblaciones rurales en Uruguay, y en América Latina en general, son portadoras de formas culturales propias que las caracterizan como productoras de espacios y territorios singulares. Paso Centurión cuenta con una historia de trabajo asociativo como forma de enfrentar y solucionar las dificultades en la producción, la salud, la educación, el transporte y otras problemáticas por las cuales la comunidad se organiza para salir adelante.

El trabajo asociativo familiar o comunal tiene una larga historia en la zona; experiencias que pudimos recolectar en estos años de trabajo, algunas prácticas asociativas, son llevadas a cabo cotidianamente por sus pobladores y aun no han sido visibilizadas como tales por no tener una finalidad específicamente económico-productiva. Sería interesante acompañar desde la extensión universitaria para que se realicen con frecuencia espacios de intercambio vecinal donde esas prácticas sean visibilizadas y reconocidas dentro de la comunidad. En particular, el trabajo universitario en la zona cuenta con experiencias de enorme importancia en el marco de la extensión. Desde la década del ' 50 del siglo pasado, los distintos servicios universitarios han llevado adelante experiencias que han formado en extensión a muchos profesionales universitarios y que han generado experiencias icónicas para la extensión en Uruguay.

Como universitarios, hemos tomado esas experiencias como parte del asociativismo de Paso Centurión para apoyar el trabajo futuro en esta área y rescatar de alguna forma la identidad de un territorio. Los modelos de apoyo orientados desde las políticas públicas no siempre tienen en cuenta las experiencias con las que cuenta la comunidad, y muchas veces 
la implantación de un modelo generado tiene dificultades o fracasa por falta de apropiación de sus participantes en un proyecto colectivo. La inclusión de las experiencias singulares en modelos de apoyo propuestos podría ser una variable que fortalezca estas experiencias.

\section{Referencias bibliográficas}

Castro, D. y Santos, C. (2018). Rasgos de la lógica estatal en la hegemonía progresista uruguaya. En Ouviña, H. y Thwaites Rey, M. (Comps.). Estados en disputa. Auge y fractura del ciclo de impugnación al neoliberalismo en América Latina (pp. 121-139). Buenos Aires: El Colectivo.

Chiappe, M.; Carambula, M. y Fernández, E. (2008). El campo uruguayo, una mirada desde la sociología rural. Montevideo: Depto. de Publicaciones de la Facultad de Agronomía.

Cruz, A. (2007). A Construçao do conceito de Economia Solidaria no Cone Sul. Revista de Estudios Cooperativos, 12(1), 6-27.

Domínguez, A.; Achkar, M.; Pesce, F. y Díaz, I. (2017). Las transformaciones territoriales del espacio agrario uruguayo: nuevas regionalidades. Geo UERJ, (32). doi:10.12957/geouerj.2018.28973

González Fajardo, D. (2016). Fortalecimiento Institucional de Procesos Asociativos Rurales en Uruguay. Modelos de Desarrollo, Grupalidad y Organizaciones del Trabajo. Psicología Política, 16(37), 349-365.

Griffin, E. (1972). Agricultural land use in Uruguay. Tesis de Ph.D. inédita. Michigan State University.

Haesbaert, R. (2004). O mito da desterritorializaçao: do "fim dos territórios" á multiterritiralidade. Rio de Janeiro: Bertrand Brasil.

Instituto Nacional de Estadística (2011). Población por área y sexo, según grupo quinquenal de edades. Total país. Recuperado de: http://ine.gub.uy/web/guest/censos-2011

Isola, G. y Martí, J.P. (2015). El significado y el proceso del cooperativismo uruguayo a treinta años del trabajo de Juan Pablo Terra. Recuperado de: https://docplayer.es/35548763-El-significado-y-el-proceso-del-cooperativismo-uruguayo-a-treinta-anos-del-trabajo-de-juan-pablo-terra-1.html

Mendivil, R. (2009). Grupos asociativos de turismo rural en Argentina. El caso de los grupos Cambio Rural del centro Regional Buenos Aires Sur del INTA. Tesis de Maestría inédita. Universidad de Buenos Aires.

Migliaro, A. y Picos, G. (2008). Por la Tierra y... con... ¿?: Estrategias de Intervención en los Procesos Asociativos Vinculados al Instituto Nacional de Colonización. En Leopold, L. (Org.). Crítica de la Cultura Organizacional. Claves para Cambiar la Organización del Trabajo. Montevideo: Psicolibros.

MVOTMA (s/f). ¿Qué es el SNAP? Recuperado de: http://www.mvotma.gub.uy/ambiente/conservacion-de-ecosistemas-y-biodiversidad/areas-protegidas/snap

Nuñez, S. (2014). Disney war. Violencia territorial en la aldea global. Montevideo: Hum.

Peixoto de Albuquerque (2004a). Asociativismo. En Cattani, A.D. (Comp.). La otra economía (pp. 31-38). Buenos Aires: Altamira-UNGS.

(2004b). Autogestión. En Cattani, A.D. (Comp.). La otra economía (pp. 39-46). Buenos Aires: Altamira-UNGS.

Pichon-Rivière, E. (2010). El proceso grupal. Recuperado de: https://eva.udelar.edu.uy/pluginfile.php/489952/ mod_resource/content/1/El\%20Proceso\%20Grupal_EPR.pdf Consultado en marzo de 2019

Piñeiro, D. (2004). Movimientos Sociales, Gobernanza Ambiental y Desarrollo Territorial Rural. Montevideo: RIMISP.

Riviere, A (1984). La psicología de Vygotski: sobre la larga proyección de una corta biografía. Infancia y Aprendizaje, (27), 7-86. 
Sarachu, G. (2009). Prácticas integrales hacia la construcción colectiva de viabilidades: Un análisis de las realizaciones y desafíos de la Incubadora de Emprendimientos Económicos Asociativos Populares (INCOOP/ UEC). Estudios Cooperativos, 14(1). 106-117.

SNAP (2018). Borrador de propuesta de ingreso de Paso Centurión y Sierra de los Ríos. Documento inédito. Szmulewicz, P.; Gutiérrez, C. y Winkler, K. (2012). Asociatividad y Agroturismo. Evaluación de las habilidades asociativas en redes de Agroturismo del sur de Chile. Estudios y Perspectivas en Turismo, (21), 1013-1034.

Terra, J. (1986). Proceso y Significado del Cooperativismo Uruguayo. Montevideo: Banda Oriental.

Tommasino, H. (2011). El desarrollo rural como un proceso de construcción de una sociedad más justa. En Proyecto Uruguay Rural 2005-2011 Evaluación de Resultados y Percepciones de los Involucrados. Vol. I. Montevideo: MGAP.

UdelaR (s/f). Convocatoria concursable a Proyectos Estudiantiles de Extensión Universitaria 2020. Recuperado de: http://www.extension.udelar.edu.uy/convocatoria-concursable-a-proyectos-estudiantiles-de-extension-universitaria-2020

UdelaR-CSEAM (2019). Sector Cooperativismo y Economía Social y Solidaria (SCES). Recuperado de: http:// www.extension.udelar.edu.uy/sces/ 